\title{
Coracoclavicular Space Widening on Radiographs After Arthroscopic Stabilization With Suspensory Fixation Does Not Affect Athletic Performance
}

\author{
Amr Ibrahim, M.D., Saleh Gameel, M.D., Tarek Mohamed Ghandour, M.D., and \\ Begad M. Samy Abbas, M.D.
}

\begin{abstract}
Purpose: To evaluate the effect of the coracoclavicular distance widening in athletes after arthroscopic acromioclavicular (AC) stabilization using a suspensory button fixation device in terms of function, athletic performance, or isokinetic assessment. Methods: Sixty-eight athletes with a minimum 6-month follow-up after AC stabilization using suspensory button fixation were allocated in 2 groups, the widening group and non-widening group, according to the measured coracoclavicular distance after 6 months from the operation. The assessment was done every 6 months by Subjective Patient Outcome for Return to Sports (SPORTS) score, Athletic Shoulder Outcome Scoring System (ASOSS), Disabilities of the Arm, Shoulder, and Hand (DASH) score, Constant-Murley score (CMS), and the coracoclavicular distance. Isokinetic testing was performed at 24 months postoperatively to evaluate shoulder abduction and external rotation strength. Results: No statistically significant differences were found between the 2 groups in terms of the DASH, ASOSS, SPORTS, and the CMS, in addition to the isokinetic testing $(P>.05)$. A statistically significant improvement in both groups over the follow-up stage was identified in the DASH, ASOSS, SPORTS, and the CMS $(P<.05)$. Conclusions: Coracoclavicular distance widening following arthroscopic suspensory button fixation for AC joint dislocation did not affect function, athletic performance, or isokinetic evaluation in athletes. Level of Evidence: III; nonrandomized, comparative trial.
\end{abstract}

A cromioclavicular (AC) joint dislocations are a common injury that account for almost $50 \%$ of sports-related shoulder injuries. ${ }^{1-3}$ The Rockwood classification is the most commonly used classification system. Conservative management is widely used for type I and II dislocations, whereas surgical treatment the intervention of choice for type IV to VI dislocations. , 4-6 $^{\text {, }}$

Many surgical options have been reported in the literature, all of which aim for full recovery with the return to the preinjury level of activity and sport

From the Orthopedic Department (A.I., S.G., T.M.G.) and Physical Medicine (B.M.S.A.), Faculty of Medicine, Ain Shams University, Cairo, Egypt.

The authors report that they have no conflicts of interest in the authorship and publication of this article. Full ICMJE author disclosure forms are available for this article online, as supplementary material.

Received September 12, 2020; accepted January 15, 2021.

Address correspondence to Tarek Mohamed Ghandour, M.D., Orthopedic Department, Faculty of Medicine, Ain Shams University, Cairo, Egypt. E-mail:tarekghndr@yahoo.com

(C) 2021 THE AUTHORS. Published by Elsevier Inc. on behalf of the Arthroscopy Association of North America. This is an open access article under the CC BY license (http://creativecommons.org/licenses/by/4.0/).

2666-061X/201495

https://doi.org/10.1016/j.asmr.2021.01.005 participation..$^{6-8}$ The suspensory button fixation was reported initially for the fixation of syndesmotic ankle injuries. The use of these devices in the treatment of AC dislocations has been described in open and arthroscopic techniques. ${ }^{7,9-11}$

The loss of AC joint reduction, as evident by coracoclavicular (CC) distance widening, is commonly reported after AC stabilization using suspensory button fixation devices. The effect of such findings on the athletic performance of patients is not yet fully understood. ${ }^{7,9,11-15}$ The purpose of this study is to evaluate the effect of CC distance widening in athletes after arthroscopic AC joint stabilization using a suspensory bottom fixation device in terms of function, athletic performance, and/or isokinetic assessment. We hypothesized that the CC distance rewidening after AC joint stabilization using a suspensory button fixation might affect the function and athletic performance of the shoulder joint.

\section{Methods}

The ethical committee approved this prospective study on March 2011. Between April 2011 and March 2016, we recruited 68 patients in this nonrandomized prospective cohort study (Fig 1), which was conducted 


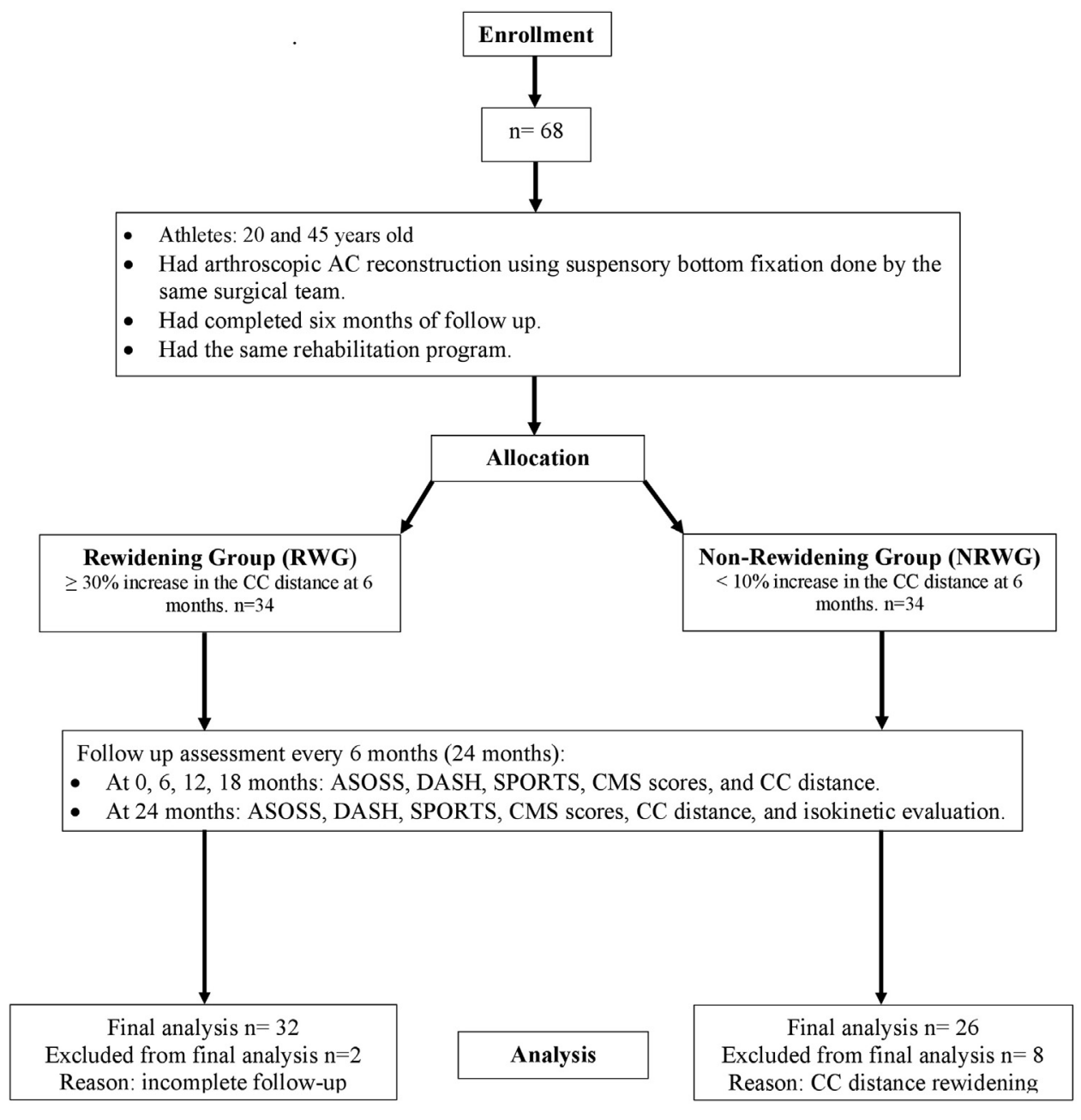

Fig 1. Study flowchart. (AC, acormioclavicular, ASOSS, Athletic Shoulder Outcome Scoring System; CC, Coracoclavicular; CMS, Constant-Murley Score; DASH, Disabilities of Arm, Shoulder and Hand; SPORTS, Subjective Patient Outcome for Return to Sports.)

at our institute. Eligible patients were atheletes aged between 20 and 45 years who had arthroscopic AC reconstruction using suspensory button fixation using the same suspensory button fixation device for acute AC dislocation Rockwood type III or V done within 1 month of the injury by our surgical team, using the same surgical technique described in our previous studies. ${ }^{7,16}$ All patients must have completed 6 months after the fixation and had the same rehabilitation program, which was formulated in 4 phases over 6 months, including (1) immobilization phase (weeks 0$6)$ : during this phase, all patients were immobilized in a pouch arm sling; (2) intermediate phase (weeks 7-12): during this phase, active assisted and active range of motion were initiated along with stretching exercises; (3) strengthening phase (weeks 13-16); during this phase, strengthening exercises including isotonic strength activities were initiated; and (4) return to activity phase (months 4-6); during this phase, strength, balance and proprioception exercises, and sports-specific activities were started.
Subjects were included in the widening group (WG) if they had a $30 \%$ or more increase in the CC distance on the 6-month-follow up radiograph when compared with the immediate postoperative radiograph, which was collected retrospectively from the patients' files. The non-widening group (NWG) included subjects with no or less than $10 \%$ increase in the CC distance at the same follow-up point. The CC distance was measured and recorded by 2 independent radiographers. To measure the CC distance, we used an anteroposterior view of the shoulder with AC projection (the beam was centered over the AC joint with $10^{\circ}$ cephalad) that was done by the same technician. Seventeen subjects (10 in WG and 7 in NWG) were also participating in another study that was published in 2019. .9-11 $^{\text {. }}$

Subjects were excluded from the study if they were outside the age range, nonathletes, noncompliant to the rehabilitation program as seen retrospectively from their files, or if there was an increase in the CC distance of $10 \%$ or more but less than $30 \%$ of their immediate postoperative radiograph. Subjects also were excluded 
if they had any associated pathology of the shoulder, such as rotator cuff tear, shoulder instability, adhesive capsulitis, fracture or dislocation of the shoulder girdle, or previous shoulder surgery.

We considered patients as athletes if they had at least 2 training sessions each week. We classified the patients according to their level of sports participation in 3 grades: grade 1: professional athletes who consider sport as their primary source of income; grade 2: amateur athletes who had at least 3 training sessions per week; and grade 3: amateur athletes who had only 2 training sessions per week. Different types of sports were categorized into 4 subtypes, according to Allain et al. ${ }^{17}$ We included athletes from type III (swimming the crawl and tennis) and type IV (basketball, volleyball, handball, and karate).

The starting point of the study, at which the recruitment occurred, was 6 months from the operation. Participant were only recruited if they fulfilled the inclusion criteria. At recruitment, each subject was given a code; the code key was kept with the code keeper, who was nonmedical personnel. A baseline assessment was done for all the study subjects using (1) the Subjective Patient Outcome for Return to Sports (SPORTS) score $^{18}$; (2) Athletic Shoulder Outcome Scoring System $(\text { ASOSS })^{19}$; (3) Disabilities of the Arm, Shoulder, and Hand (DASH) score se,21 $^{2}$ (4) Constant-Murley score (CMS), which was done by a single experienced examiner in shoulder surgery; and (5) CC distance in the AC joint projection, which was measured and recorded by 2 independent radiographers to avoid intra- and interobserver bias. This baseline assessment was performed in all subjects at the time of recruitment, keeping in mind that subjects were recruited only if a 6month duration had passed since the operation as a part of the inclusion criteria. All subjects in both groups were assessed every 6 months for 24 months using the aforementioned parameters.

\section{Isokinetic Evaluation}

A single examiner (a physical medicine physician, M.D.) performed an isokinetic muscle strength evaluation for the shoulder external rotation and abduction in all patients at 24-month follow-up using the isokinetic Biodex System 3 (Biodex Medical Systems, Shirley, NY).

For assessment of external shoulder rotation, patients were positioned in a seated position with $45^{\circ}$ of abduction in the scapular plane and $90^{\circ}$ of elbow flexion. The rotational axis was aligned with the humeral shaft axis. Subjects were stabilized with straps, as described in the manufacturer's manual.

For assessment of shoulder abduction, patients were positioned in a seated position with full elbow extension and full shoulder adduction as the starting position. The rotational axis was aligned with the axis of the AC joint. The nonoperated side was tested at the beginning to familiarize the patient with the procedure and was not included in the final analysis.

The test protocol consisted of concentric contractions at 3 angular speeds, $60^{\circ}$ per second and $120^{\circ}$ per second for 5 repetitions, and $180^{\circ}$ per second for 10 repetitions. The test protocol was thoroughly explained to patients. A warm-up cycle preceded the initiation of the procedure, which consisted of a 5-minute upper body cycle of one maximal effort and three submaximal efforts.

Visual feedback was given, but not verbal encouragement. The normalized torque per body weight in Newton-meters per kilogram was measured. The results were collected, tabulated, and kept with the code keeper.

\section{Blinding}

The clinical examiner, the 2 radiographers, and the physical medicine specialist were all blinded to the group allocation. The code keeper, who was nonmedical personnel, was responsible for keeping the study code key, the completed DASH, ASOSS, and SPORTS questionnaires, the CMS results, the radiographer's reports. He was the only permitted researcher to approach for the patients by phone to schedule followup appointments and radiographic evaluations. The code keeper was not involved in the clinical assessment of patients.

\section{Confounders}

As randomization was not possible in this study, we tried to control for possible confounders at the level of the study design by restriction and matching. Patients who were handled by different surgical teams or had different fixation methods, different follow-up duration, or were practicing extreme sports (martial arts, wrestling, or climbing, etc.) were not included in the study. We did not consider karate as an extreme sport, as we included 2 amateur karate players, one in each group. In addition, subjects in the NWG were matched to those in the WG in respect of age, sports type, and level of participation.

\section{Sample Size}

We performed a 2-tailed a priori test evaluation for the needed sample size, to achieve power $(1-b)$ of $80 \%$ when alpha $=0.05$, using the $G^{*}$ Power software (version 3.1.9.6; Heinrich-Heine-Universität, Düsseldorf, Germany). The assessment was done for the CMS and the isokinetic evaluation of the shoulder abduction.

We assumed a mean CMS difference of 16 points between the 2 groups and a standard deviation of 20 points; 26 subjects had to be recruited in each group. For the isokinetic assessment of the shoulder abduction, we assumed a mean difference of 0.05 and a standard deviation of 0.06 Newton-meters per kilogram, 24 
Table 1. Study Demographics

\begin{tabular}{lcc}
\hline & $\begin{array}{c}\text { Widening } \\
\text { Group (WG) }\end{array}$ & $\begin{array}{c}\text { Non-widening } \\
\text { Group (NWG) }\end{array}$ \\
\hline Age, y & $32.7 \pm 8.48$ & $35.6 \pm 7.43$ \\
Sex & 22 & 17 \\
Male & 10 & 9 \\
Female & & 14 \\
Laterality & 15 & 12 \\
Right & 17 & 6 \\
Left & & 18 \\
Sport type & 3 & 8 \\
Basketball & 20 & 9 \\
Volleyball & 9 & 2 \\
Handball & 10 & 1 \\
Swimming & 8 & 3 \\
Tennis & 1 & 10 \\
Karate & & 13 \\
Participation Level & 3 & \\
Professional & 21 & \\
Amateur: 3 sessions weekly & 8 & \\
Amateur: 2 sessions weekly & & \\
\hline & & \\
\hline
\end{tabular}

subjects had to be included in each group. Our target was to include 34 cases in each group in an attempt to compensate for lost study subjects during the follow-up.

\section{Statistics}

We used the SPSS software (version 23.0; IBM, Armonk, NY) in the analysis of data. We calculated the range, mean, and standard deviation. The $\chi^{2}$ test was used to assess the statistically significant difference between the 2 groups in respect of the nominal variables. The intraclass correlation coefficient was calculated to measure the reliability of the readings of the 2 radiographers.

Age, sex, laterality, and type of sport and level of participation were identified as possible confounders. The multivariate analysis of covariance (MANCOVA) was conducted to test the effect of group allocation on the dependent variables while controlling for the age, sex, laterality, type of sport, and the level of participation. Before we conducted the MANCOVA test, data were tested for normality using the Kolmogorov-Smirnov test, homogeneity of variances and covariances using the Levene test, and homogeneity of regression slopes.

If the results of the MANCOVA test showed an insignificant effect of the suspected confounder, the independent sample $t$ test was used to test numerical variables for statistically significant difference, and to calculate the mean difference and the $95 \%$ confidence interval of the mean difference.

When variables were measured for more than one occasion during the study, the 2-way repeatedmeasures analysis of variance (ANOVA) was used to detect statistically significant changes within the same group and to assess for any differences in the changes in relation to the time between the 2 groups.
Table 2. The ICC for the Reliability of the CC Distance Measurements

\begin{tabular}{lccc}
\hline & & \multicolumn{2}{c}{ Confidence Interval } \\
\cline { 3 - 4 } & ICC & Lower & Upper \\
\hline CC pre & 0.87 & 0.79 & 0.92 \\
CC 0 & 0.89 & 0.82 & 0.94 \\
CC 6 & 0.89 & 0.81 & 0.93 \\
CC 12 & 0.93 & 0.88 & 0.95 \\
CC 18 & 0.88 & 0.85 & 0.90 \\
CC 24 & 0.86 & 0.84 & 0.88
\end{tabular}

NOTE. 0 indicates baseline assessment; 6, 6-month assessment; 12, 12-month assessment; 18, 18-month assessment; and 24, 24-month assessment.

CC, coracoclavicular; ICC, intraclass correlation coefficient.

Statistical significance was evaluated by the $95 \%$ confidence interval and the 2-tailed significance ( $P$ value). We rejected the null hypothesis if $95 \%$ confidence interval was narrow and did not include the zero value, in addition to a $P$ value of $<.05$. A Bonferroni correction of $P$ value was considered when multiple comparisons were used in the MANCOVA test; a statistically significant difference was considered if $P$ value was $<.03$.

A minimal clinically important difference of 10.4 points for the CMS and 10.2 points for the DASH score was considered as cut points to consider a change in these scores as clinically significant. ${ }^{22,23}$

\section{Results}

Sixty-eight subjects were recruited for this study. Thirty-four subjects were allocated in each group according to their CC distance. Only 58 patients were eligible for the final analysis (32 in the WG, and 26 in the NWG). Two cases from the WG were excluded from the final analysis for incomplete follow-up data due to noncompliance. In comparison, 8 cases from NWG were excluded from the final analysis, as CC distance widening occurred during the study course and after their recruitment.

The $\chi^{2}$ test revealed that there were no statistically significant differences between the 2 groups in terms of age, sex, laterality, sport type, or level of participation (Table 1). The intraclass correlation coefficient showed an acceptable reliability of the CC distance measurements between the 2 radiographers (Table 2).

Table 3. Effect of Confounders on the Statistical Difference Between the 2 Groups, Wilk's Lambda test, MANCOVA

\begin{tabular}{lccc}
\hline & F Value & $P$ & Effect Size \\
\hline Age & 1.38 & .222 & .690 \\
Sex & 1.04 & .473 & .625 \\
Laterality & .96 & .551 & .606 \\
Sport type & 1.07 & .440 & .633 \\
Participation level & .57 & .923 & .478 \\
\hline
\end{tabular}

MANCOVA, multivariate analysis of covariance. 
Table 4. The Independent Sample $t$ Test

\begin{tabular}{|c|c|c|c|c|c|c|c|c|c|c|}
\hline \multirow[b]{3}{*}{ DASH 0 } & & & & & & & \multirow[b]{2}{*}{ Mean Difference } & \multicolumn{2}{|c|}{$\begin{array}{l}95 \% \text { Confidence } \\
\text { Interval of the } \\
\text { Mean Difference }\end{array}$} & \multirow[b]{2}{*}{$P$ Value } \\
\hline & \multicolumn{3}{|c|}{ Widening Group (WG) } & \multicolumn{3}{|c|}{ Non-widening Group (NWG) } & & Lower & Upper & \\
\hline & 44.5 & \pm & 2.9 & 44.8 & \pm & 1.8 & -3.8 & -1.7 & .95 & .568 \\
\hline DASH 6 & 37.0 & \pm & 2.1 & 36.3 & \pm & 1.2 & .64 & -.29 & 1.5 & .174 \\
\hline DASH 12 & 31.2 & \pm & 2.5 & 30.2 & \pm & 1.3 & 1.0 & -.09 & 2.1 & .073 \\
\hline DASH 18 & 24.1 & \pm & 2.5 & 23.0 & \pm & 1.5 & 1.0 & -.09 & 2.1 & .072 \\
\hline DASH 24 & 14.9 & \pm & 2.2 & 13.7 & \pm & 2.7 & 1.1 & -.18 & 2.4 & .089 \\
\hline CMS 0 & 64.2 & \pm & 4.1 & 67.1 & \pm & 6.5 & -2.8 & -5.7 & -.03 & .066 \\
\hline CMS 6 & 75.7 & \pm & 3.6 & 77.0 & \pm & 3.4 & -1.3 & -3.2 & .5 & .155 \\
\hline CMS 12 & 83.1 & \pm & 4.1 & 85.0 & \pm & 4.2 & -1.9 & -4.1 & .3 & .090 \\
\hline CMS 18 & 85.9 & \pm & 3.4 & 87.5 & \pm & 3.2 & -1.6 & -3.4 & .1 & .066 \\
\hline CMS 24 & 85.5 & \pm & 3.7 & 87.1 & \pm & 3.4 & -1.5 & -3.4 & .3 & .097 \\
\hline ASOSS 0 & 42.6 & \pm & 1.4 & 43.2 & \pm & 1.2 & -.60 & -1.3 & .13 & .107 \\
\hline ASOSS 6 & 49.8 & \pm & 2.9 & 50.8 & \pm & 1.9 & -1.0 & -2.3 & .31 & .130 \\
\hline ASOSS 12 & 57.0 & \pm & 2.8 & 58.2 & \pm & 1.5 & -1.1 & -2.4 & .06 & .064 \\
\hline ASOSS 18 & 66.7 & \pm & 4.7 & 68.5 & \pm & 1.9 & -1.7 & -3.7 & .19 & .076 \\
\hline ASOSS 24 & 78.7 & \pm & 4.7 & 81.1 & \pm & 5.8 & -2.4 & -5.2 & .30 & .080 \\
\hline SPORTS 0 & 3.0 & \pm & .5 & 3.3 & \pm & .97 & -.25 & -.65 & .15 & .215 \\
\hline SPORTS 6 & 3.2 & \pm & .8 & 3.5 & \pm & 1.2 & -.29 & -.84 & .25 & .287 \\
\hline SPORTS 12 & 3.9 & \pm & 1.4 & 4.2 & \pm & 1.5 & -.33 & -1.1 & .43 & .392 \\
\hline SPORTS 18 & 5.8 & \pm & 2.2 & 6.3 & \pm & 2.7 & -.46 & -1.7 & .85 & .484 \\
\hline SPORTS 24 & 6.1 & \pm & 2.3 & 6.3 & \pm & 2.7 & -.18 & -1.5 & 1.1 & .785 \\
\hline CC post & 10.3 & \pm & 1.0 & 10.4 & \pm & 1.0 & -.14 & -.70 & .40 & .592 \\
\hline CC 0 & 14.9 & \pm & .8 & 12.0 & \pm & 1.8 & 2.8 & 2.1 & 3.5 & .000 \\
\hline CC 6 & 15.8 & \pm & 1.5 & 12.4 & \pm & 2.2 & 3.4 & 2.4 & 4.4 & .000 \\
\hline CC 12 & 15.6 & \pm & 1.3 & 12.3 & \pm & 2.0 & 3.2 & 2.3 & 4.1 & .000 \\
\hline CC 18 & 15.6 & \pm & 1.3 & 12.2 & \pm & 1.9 & 3.4 & 2.5 & 4.3 & .000 \\
\hline CC 24 & 15.7 & \pm & 1.3 & 12.2 & \pm & 2.0 & 3.5 & 2.6 & 4.4 & .000 \\
\hline External rotation $60^{\circ}$ & .43 & \pm & .02 & .45 & \pm & .03 & -.01 & -.03 & .001 & .067 \\
\hline External rotation $120^{\circ}$ & .40 & \pm & .02 & .41 & \pm & .04 & -.01 & -.03 & .001 & .072 \\
\hline External rotation $180^{\circ}$ & .36 & \pm & .02 & .38 & \pm & .03 & -.01 & -.03 & .001 & .083 \\
\hline Abduction $60^{\circ}$ & .73 & \pm & .01 & .74 & \pm & .01 & .005 & -.01 & .001 & .074 \\
\hline Abduction $120^{\circ}$ & .71 & \pm & .01 & .72 & \pm & .01 & .006 & -.01 & .001 & .071 \\
\hline
\end{tabular}

NOTE. 0 indicates baseline assessment; 6, 6-month assessment; 12, 12-month assessment; 18, 18-month assessment; and 24, 24-month assessment.

ASOSS, Athletic Shoulder Outcome Scoring System; CC, Coracoclavicular; CMS, Constant-Murley Score; DASH, Disabilities of Arm, Shoulder and Hand; Post, immediately postoperative; SPORTS, Subjective Patient Outcome for Return to Sports.

The MANCOVA showed that there was no statistically significant effect of the age, sex, laterality, sports type, or level of participation on the results of the DASH, CMS, ASOSS, or SPORTS scores, $P>.05$. In addition, there was no statistically significant effect on the changes in the $\mathrm{CC}$ distance on the isokinetic muscle testing, $P>.05$ (Table 3).

Although the means of the DASH, CMS, and ASOSS scores, in addition to the isokinetic assessments, were greater in the NWG than in the WG, the independent sample $t$ test showed that this difference was not statistically significant: in respect of the DASH score (at the recruitment; $P=.568$, at 6 -month follow-up; $P=.174$, at 12 -month follow-up; $P=.073$, at 18 month follow-up; $P=.072$, at 24-month follow-up; $P=.089$ ), in respect of the CMS score (at the recruitment; $P=.066$, at 6 -month follow-up; $P=.155$, at 12 -month follow-up; $P=.090$, at 18-month followup; $P=.066$, at 24 -month follow-up; $P=.097$ ); in respect of the ASOSS score (at the recruitment; $P=$ .107 , at 6 -month follow-up; $P=.130$, at 12 -month follow-up; $P=.064$, at 18 -month follow-up; $P=$ .076 , at 24-month follow-up; $P=.080)$; in respect of the SPORTS score (at the recruitment; $P=.215$, at 6month follow-up; $P=.287$, at 12 -month follow-up; $P=.392$, at 18 -month follow-up; $P=.484$, at 24 month follow-up; $P=.785)$; in respect of the isokinetic assessments (external rotation at $60^{\circ}$ /seconds of angular velocity; $P=.067$, external rotation at $120^{\circ} \mathrm{I}$ seconds of angular velocity; $P=.072$, external rotation at $180^{\circ}$ /seconds of angular velocity; $P=.083$, abduction at $60^{\circ}$ /seconds of angular velocity; $P=.074$, abduction at $120^{\circ} /$ seconds of angular velocity; $P=$ .071 , abduction at $180^{\circ}$ /seconds of angular velocity; $P=.064)$. In addition, the $95 \%$ confidence intervals of the mean differences were wide and contained the zero value; therefore, we failed to reject the null hypothesis (Table 4). 


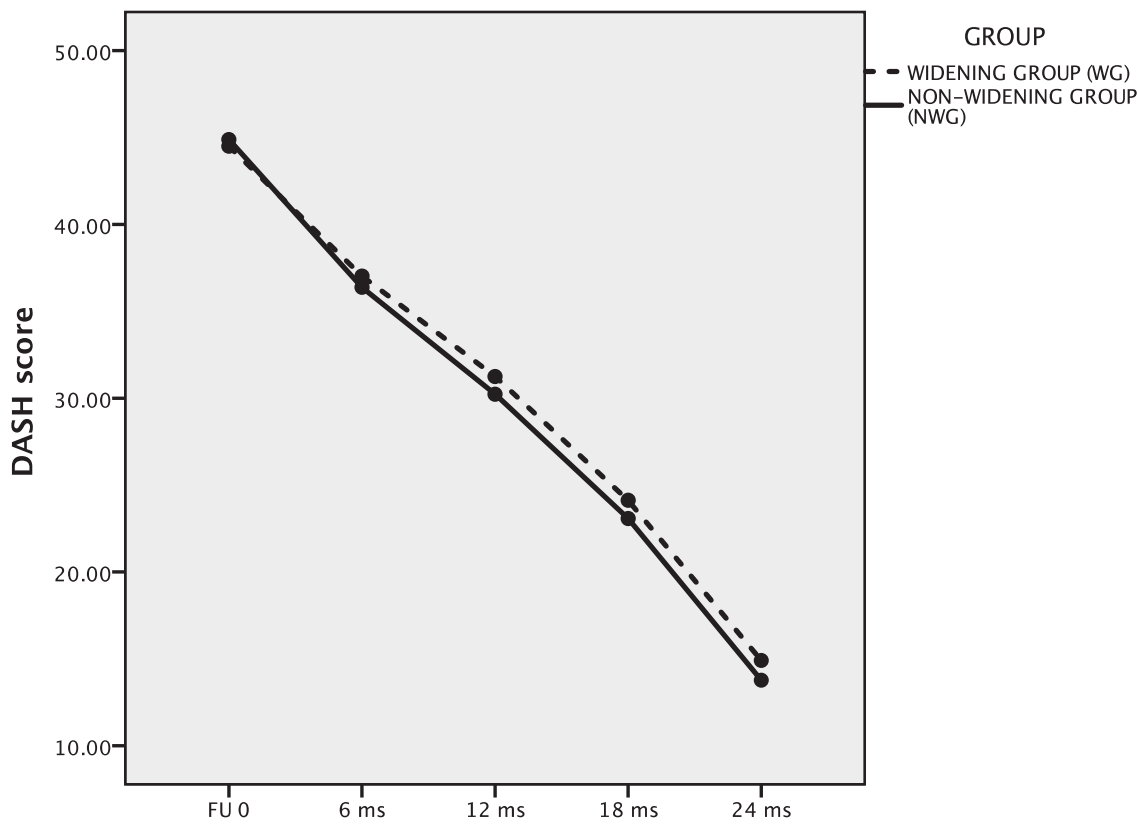

Fig 2. Changes in DASH score in relation to time in both groups. The graph shows improvement of the DASH score in both groups during the follow-up phase. (DASH, Disabilities of Arm, Shoulder and Hand.)

The 2-way repeated-measures ANOVA yielded a significant improvement over the study course in the DASH, CMS, ASOSS, and SPORTS scores of each group (for DASH score; $P=.515$, for CMS score; $P=.790$, for ASOSS score; $P=.663$, for SPORTS score; $P=.635$ ). Therefore, the null hypothesis that there were no changes was rejected. The changes over time comparison did not show any significant differences between the 2 groups $(P>.05)$. As expected, the 2-way repeated-measures ANOVA yielded a significant increase in the CC distance of the WG over the study course $(P<.001)$, and nonsignificant changes in the NWG, $(P>.05)$ (Figs 2-6).

In terms of clinical significance, all study subjects achieved the minimal clinically important difference for the CMS and the DASH scores from the initial stage to the final follow-up after 2 years.

\section{Discussion}

We did not find statistically significant differences between the 2 groups in terms of function, athletic performance, or isokinetic assessment. We used the
Fig 3. Changes in CMS in relation to time in both groups. The graph shows improvement in the CMS score in both groups during the follow-up phase. (CMS, Constant-Murley Score.)

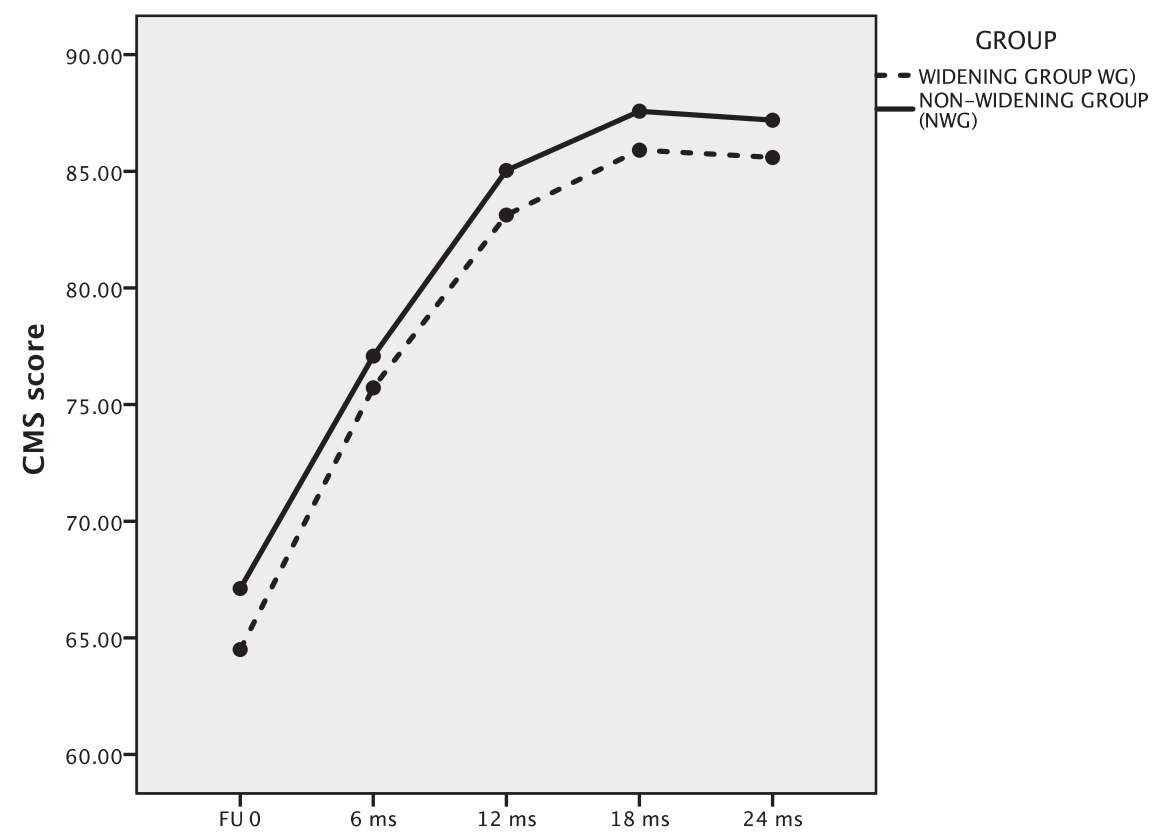


Fig 4. Changes in ASOSS in relation to time in both groups. The graph shows improvement in the ASOSS score in both groups during the follow up phase. (ASOSS, Athletic Shoulder Outcome Scoring System.)

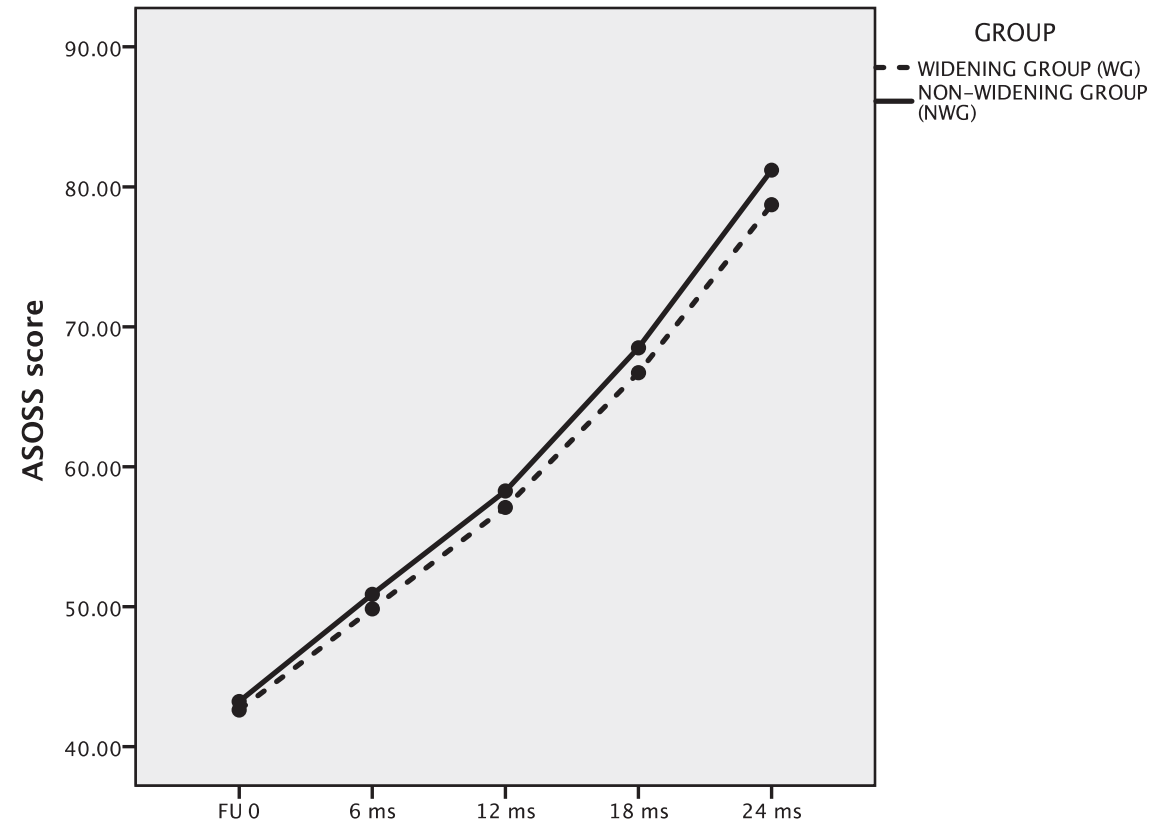

isokinetic testing as an objective tool to assess the shoulder function after suspensory button fixation of the AC joint reconstruction.

The loss of reduction after AC reconstruction using suspensory fixation devices has been reported frequently in the literature. ${ }^{7,9,11,13,14,16,24,25}$ While many researchers attempted to investigate the athletic performance following AC reconstruction, the effect of loss of reduction of the AC joint on the athletic performance of the patients was not reported in the literature before. $8,19,26,27$

In a systematic review done by Verstift et al., ${ }^{19}$ they reviewed 12 articles that evaluated the return to the preinjury level of sport after a repair of Rockwood types III to VI dislocations, with a total number of patients of 498, of which 462 patients were engaged in sports activities. They concluded that the return to sport participation after this kind of surgery is high.

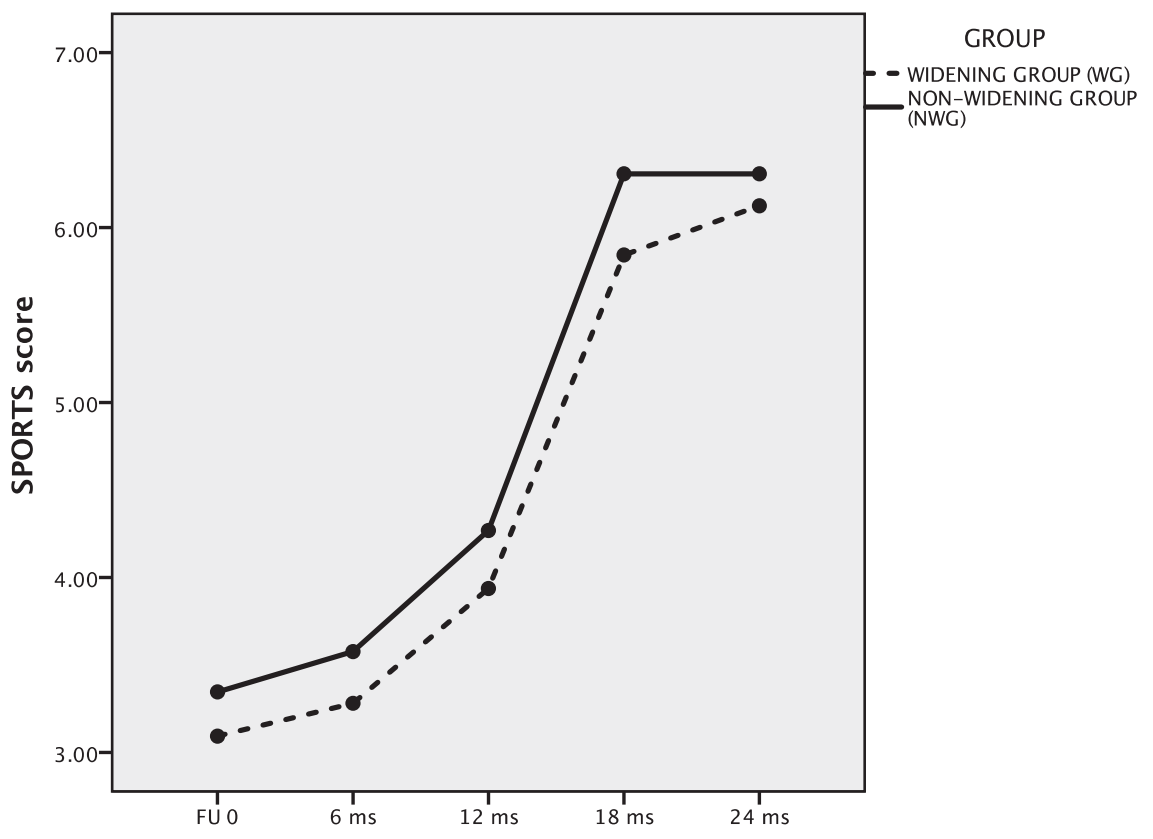

Fig 5. Changes in SPORTS in relation to time in both groups. The graph shows improvement in the SPORTS score in both groups during the follow-up phase. (SPORTS, Subjective Patient Outcome for Return to Sports.) 


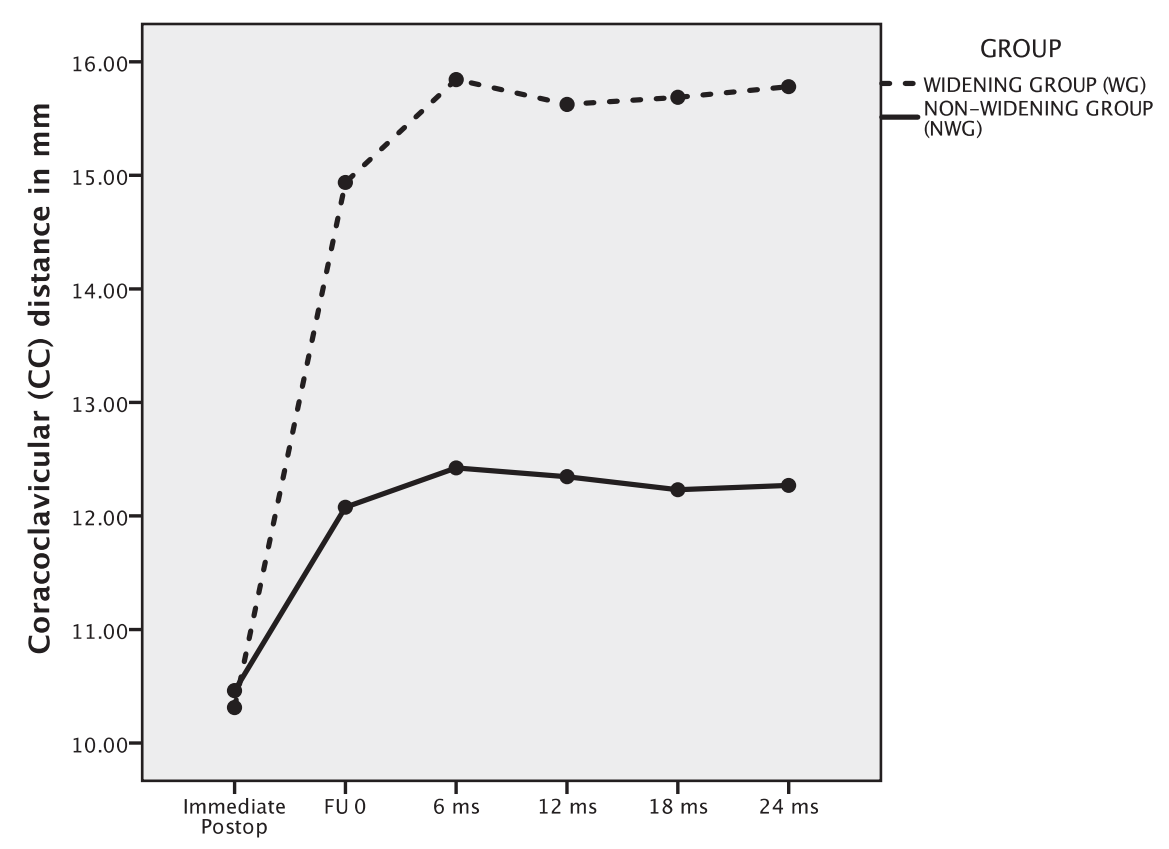

Fig 6. Changes in the $\mathrm{CC}$ distance in relation to time in both groups. The graph shows widening of the CC distance in the WG during the follow-up phase. (CC, coracoclavicular.)
In another systematic review by Kay et al., ${ }^{27} 315$ patients, who had a surgical intervention for AC dislocation, were included in their reviewed articles. They concluded an excellent rate of sport participation after surgical reconstruction of the dislocated AC joint.

In addition, Saier et al. ${ }^{15}$ evaluated the return to activity level in 42 subjects after arthroscopically assisted reconstruction of acutely dislocated AC joint using suspensory button fixation. In their study, all included subjects were able to participate in sporting activities after the surgery. There was no observed difference in participation for those who practice contact or overhead sports.

In our study, all our 58 subjects returned to sport participation. Although we had greater results in the NWG in terms of function (CMS and DASH scores), athletic performance (ASOSS score), and the objective shoulder muscle strength (isokinetic testing), this difference was not statistically significant. This insignificant difference might have happened since we adjusted our sample size calculation to detect a power of $80 \%$. A follow-up study with a greater power value, and eventually, a larger sample size might be needed to confirm any significant difference.

\section{Limitations}

We had some limitations in our study; due to the nature of the study, we used a nonrandomized design, which might have increased the risk for confounders. We failed to reject the null hypothesis, even though we had greater results in the NWG. The definition of loss of reduction was based on a single point measurement at the time of recruitment. We defined widening as an increase of the CC distance of $30 \%$ or more, and non-widening as an increase of less than $10 \%$ at the time of recruitment. We excluded those with an increase of $10 \%$ to $30 \%$ to avoid this gray zone. During the follow-up phase, we had to exclude 8 subjects from the NWG due to a late-onset loss of reduction, which might have affected our final analysis.

\section{Conclusions}

CC distance widening following arthroscopic suspensory button fixation for AC dislocation did not affect function, athletic performance, or isokinetic evaluation in athletes.

\section{References}

1. Lee S, Bedi A. Shoulder acromioclavicular joint reconstruction options and outcomes. Curr Rev Musculoskelet Med 2016;9:368-377.

2. Mori D, Yamashita F, Kizaki K, Funakoshi N, Mizuno Y, Kobayashi M. Anatomic coracoclavicular ligament reconstruction for the treatment of acute acromioclavicular joint dislocation: Minimum 10-year follow-up. JB JS Open Access 2017;2:e0007.

3. Boffano M, Mortera S, Wafa H, Piana R. The surgical treatment of acromioclavicular joint injuries. EFORT Open Rev 2017;2:432-437.d.

4. Song T, Yan X, Ye T. Comparison of the outcome of early and delayed surgical treatment of complete acromioclavicular joint dislocation. Knee Surg Sport Traumatol Arthrosc 2016;24:1943-1950.

5. Babhulkar A, Pawaskar A. Acromioclavicular joint dislocations. Curr Rev Musculoskelet Med 2014;7:33-39. 
6. Ulucay C, Ozler T, Akman B. Treatment of acromioclavicular joint injuries in athletes and in young active patients. J Trauma Treat 2016;05.

7. Abdelrahman AA, Ibrahim A, Abdelghaffar K, Ghandour TM, Eldib D. Open versus modified arthroscopic treatment of acute acromioclavicular dislocation using a single tight rope: Randomized comparative study of clinical outcome and cost-effectiveness. J Shoulder Elbow Surg 2019;28:2090-2097.

8. Porschke F, Schnetzke M, Aytac S, Studier S, Alfred P, Thorsten G. Sports activity after anatomic acromioclavicular joint stabilisation with flip-button technique. Knee Surg Sport Traumatol Arthrosc 2017;25:1995-2003.

9. Darabos N, Vlahovic I, Gusic N, Darabos A, Bakota B, Miklic D. Is AC TightRope fixation better than Bosworth screw fixation for minimally invasive operative treatment of Rockwood III AC joint injury? Injury 2015;46: S113-S118.

10. Jensen G, Katthagen JC, Alvarado LE, Lill H, Voigt C. Has the arthroscopically assisted reduction of acute AC joint separations with the double tight-rope technique advantages over the clavicular hook plate fixation? Knee Surg Sport Traumatol Arthrosc 2014;22:422-430.

11. Hann C, Kraus N, Minkus M, Maziak N, Scheibel M. Combined arthroscopically assisted coraco- and acromioclavicular stabilization of acute high-grade acromioclavicular joint separations. Knee Surg Sport Traumatol Arthrosc 2018;26:212-220.

12. Sun LJ, Lu D, Tao ZY, et al. Analysis of risk factors for loss of reduction after acromioclavicular joint dislocation treated with the suture-button. J Orthop Sci 2019;24:817-821.

13. Horst K, Dienstknecht T, Andruszkow H, Gradl G, Kobbe P, Pape HC. Radiographic changes in the operative treatment of acute acromioclavicular joint dislocation-TightRope technique vs. K-wire fixation. Polish J Radiol 2013;78:15-20.

14. Chaudhary D, Jain V, Joshi D, Jain JK, Goyal A, Mehta N. Arthroscopic fixation for acute acromioclavicular joint disruption using the TightRope device. J Orthop Surg (Hong Kong) 2015;23:309-314.

15. Saier T, Plath JE, Beitzel K, et al. Return-to-activity after anatomical reconstruction of acute high-grade acromioclavicular separation. BMC Musculoskelet Disord 2016:1-7.

16. Ibrahim A, Gameel S, Abdelghafar K, Ghandour TM, Samy Abbas BM. Rehabilitation posture does not affect the outcome of arthroscopically treated acromioclavicular dislocation. Arthroscopy 2020;36:2635-2641.
17. Allain J, Goutallier D, Glorion C. Long-term results of the Latarjet procedure for the treatment of anterior instability of the shoulder. J Bone Joint Surg Am 1998;80: 841-852.

18. Blonna D, Bellato E, Caranzano F, et al. Validity and reliability of the SPORTS score for shoulder instability. Joints 2014;2:59-65.

19. Verstift DE, Welsink CL, Spaans AJ, van den Bekerom MPJ. Return to sport after surgical treatment for high-grade (Rockwood III-VI) acromioclavicular dislocation. Knee Surg Sport Traumatol Arthrosc 2019;27: 3803-3812.

20. Booker S, Alfahad N, Scott M, et al. Use of scoring systems for assessing and reporting the outcome results from shoulder surgery and arthroplasty. World J Orthop 2015;6: 244-251.

21. Kirkley A, Griffin S, Dainty K. Scoring systems for the functional assessment of the shoulder. Arthroscopy 2003;19:1109-1120.

22. Harris JD, Brand JC, Cote MP, Faucett SC, Dhawan A. Research Pearls: The significance of statistics and perils of pooling. Part 1: Clinical versus statistical significance. Arthroscopy 2017;33:1102-1112.

23. Kukkonen J, Kauko T, Vahlberg T, Joukainen A, Äärimaa V. Investigating minimal clinically important difference for Constant score in patients undergoing rotator cuff surgery. J Shoulder Elbow Surg 2013;22: 1650-1655.

24. Bin Abd Razak HR, Yeo EMN, Yeo W, Lie TTD. Short-term outcomes of arthroscopic TightRope ${ }^{\circledR}$ fixation are better than hook plate fixation in acute unstable acromioclavicular joint dislocations. Eur J Orthop Surg Traumatol 2018;28:869-875.

25. Choi S, Lee TJ, Kim MK, Park JE, Kang H. Midterm results of coracoclavicular stabilization with double augmentation for acute acromioclavicular dislocation. Springerplus 2016;5:1858.

26. Müller D, Hoffmann YRR, Welsch MBF, Stein UST. Return to sport after acute acromioclavicular stabilization: A randomized control of double-suture-button system versus clavicular hook plate compared to uninjured shoulder sport athletes. Knee Surg Sport Traumatol Arthrosc 2018;26:3832-3847.

27. Kay J, Memon M, Alolabi B. Return to sport and clinical outcomes after surgical management of acromioclavicular joint dislocation: A systematic review. Arthroscopy 2018;34:2910-2924.el. 